\title{
Handwriting Recognition by Machine Learning
}

\author{
Anandika Sharma, Anupam Sharma
}

\begin{abstract}
Handwriting is one of the most natural ways of communication among people. The handwriting recognition task is the main concern of scientific community because handwriting can be varies with the same person or from one person to another hence the prediction of human behavior through handwriting is a complex task. Earlier the handwriting analysis has been done by graphologists but due to the modernization and the arrival of digital world the handwriting analysis can be done with the help of computer aided machines. Different software and algorithms has been defined to do the analysis. In the new world of machine learning handwriting recognition and the prediction of human behavior can be done by using different techniques of machine learning which increase the speed of analysis This paper studies the recent advances and the trends in the field of handwriting recognition by machine learning.
\end{abstract}

Keywords-handwriting, graphologist, machine learning.

\section{INTRODUCTION}

Handwriting is one of the most natural ways of communication among people. Every individual has different style of writing that is why people can judge the behavior of different people through handwriting. The handwriting recognition task is the main concern of scientific community because handwriting can be varies with the same person or from one person to another hence the prediction of human behavior through handwriting is a complex task.

Manuscript Received on July 22, 2019

Anandika Sharma, School of humanities and social sciences Thapar institute of engineering and technology Patiala, Punjab

Dr. Anupam Sharma, School of humanities and social sciences Thapar institute of engineering and technology Patiala, Punjab
Earlier the handwriting analysis has been done by graphologists but due to the modernization and the arrival of digital world the handwriting analysis can be done with the help of computer aided machines. Different software and algorithms has been defined to do the analysis. In the new world of machine learning handwriting recognition and the prediction of human behavior can be done by using different techniques of machine learning which increase the speed of analysis. This paper studies the recent advances and the trends in the field of handwriting recognition by machine learning methods.

Earlier the graphology method is used to evaluate and understand the handwriting pattern to predict behavior of human. But this method is time consuming and costly method. So later on the computer aided methods has been used to recognize the handwriting pattern and behavior of human. Handwriting analysis is one if the technique to predict the human personality including behavior, anger, self-esteem, honesty, fears and so on [1]. Different parameters of personality trait has been taken such as baseline, slant, margin, width and so on as the input to the machine learning methods and gives output in the form of personality trait. Detailed review of different authors has explained in this paper.

\section{OBJECTIVES}

1. To predict the human behaviour through handwriting.

2. To study the recent advances and the trends in the field of handwriting recognition by machine learning techniques.

\section{SUMMARY OF RECENT ADVANCES AND TRENDS}

Prachi desai, aayush dhavale et al (2015) [2], depicts the graph-logical technique in which the human personality can be determine by evaluating the various feature from handwriting like page margin, slant of alphabet, the baseline etc.

Here the computer aided methods can be used over graphological method to extract the feature of handwriting which helps to increase the speed and efficiency of analysis. Machine learning tool is used for the analysis and the implementation of machine learning can be based on the neural network in which baseline,

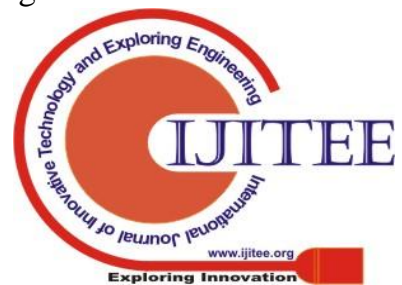




\section{HANDWRITING RECOGNITION BY MACHINE LEARNING}

letter slant, and height of $\mathrm{T}$ bar and width of margin features of handwriting are taken under consideration. The extracted features are the input to the neural network and the output gives the personality trait to the user. The author explain some algorithms which can be used for the analysis like polygonalization(used to find the slant of baseline), thresholding algorithm(transforming grayscale images into binary images), template matching( finding small parts of an image which match with the given image) and artificial neural network( predict the personality trait). In this paper the training data set comprises 100 samples, initially image processing tools is used in which the feature vector matrix find out the same handwriting samples. The classes can be generated based on the characteristic of particular trait suggested by the graphologist and then its manually mapped into its corresponding class to find out the data set, later on a supervised machine learning method is used in which KNN classifier can be used to find out the class based on similarity matrix. The similarity matrix method is used to compare the feature vector matrix of new handwriting with the trained dataset. The identified class then maps the handwriting to its corresponding trait and the result can be stores in the trained dataset. By storing the new sample into the existing class will be able to implement the machine learning that will increase the accuracy for future results.

Champa HN and Dr KR ananda kumar (2010) [3], explains to predict the human behavior through handwriting analysis by using ANN (artificial neural network). Handwriting reveals the personality of a human in various respects like emotional, fear, honest, defense etc. Previously the graphological method is used to understand the personality of human to detect the features of handwriting like baseline, size of letter, writing pressure, spacing, speed of handwriting and many more. Three parameters are taken under consideration like baseline, pen pressure and the height of the ' $t$ ' bar. Baseline in handwriting is the line along which the writing flows, the three baselines are ascending (which shows the pessimistic behavior of human), descending (which shows the optimistic behavior of human) and level (shows the normal behavior of human). The second parameter Pen pressure is the amount of pressure exerted on the paper while writing is the emotional intensity. The writer can be classified as light writer, medium writer and heavy/deep writer. And the third parameter ' $t$ ' bar which reveals accurate information about the writer, every individual has different way of writing ' $t$ ' letter so it can be classified as ' $t$ ' bar crossed very high(the person is highly self-esteem), crossed above the middle zone( moderate self- esteem), crossed very low(low self- esteem) and crossed above the stem(dreamer). The author used the methodology for the identification of certain trait through examine the ' $t$ ' letter is template matching with the certain predefined template in which the grey value of image $\mathrm{g}(\mathrm{x}, \mathrm{y})$ matched with the given image $f(x, y)$ and the decision making on the matched pattern is identified by using hamming distance. The calculated 30 values of three parameters are the input to the ANN (artificial neural network) and 30 different combination of output has been analyzed to predict the personality traits of the human. The author uses the MATLAB tool for the purpose.

Manimala, meghashree, poornima gokhale et al (2016) [4], proposed the system where the analysis of human behavior can be estimated faster than the manual system with the accuracy of $93.77 \%$. As handwriting is the impact of human behavior in which the brain print is left on the paper. The author had measured nine parameters of personality trait such as size, slant of the words, space between words, breaks in writing, pressure, margin, baseline, loop of ' $e$ ' and the distance between title(dot) and stem of 'I' . The proposed work has analyzed both micro and macro features of handwriting in which the first step is image acquisition where handwritten images having resolution of 300dpi can be taken. And at last feature extraction has been done in which the dimensionality can be reduced from a high dimensional data and the personality can be detected by examine these nine given parameters.

Table 1: Summary table of approach used by different authors

\begin{tabular}{|c|c|c|c|}
\hline $\begin{array}{l}\text { SR } \\
\text { NO }\end{array}$ & AUTHORS & $\begin{array}{lr}\text { YEAR } & \text { OF } \\
\text { PUBLICATION }\end{array}$ & $\begin{array}{l}\text { APPROACH } \\
\text { USED }\end{array}$ \\
\hline 1 & $\begin{array}{lr}\text { Prof } & \text {.Akshita } \\
\text { Chanchlani1, } & \text { Pratima } \\
\text { Kharade, } & \text { Rutuja } \\
\text { Kapase, } & \text { Sonal } \\
\text { Janvalka } & \\
\end{array}$ & 2017 & $\begin{array}{l}\text { Artificial neural } \\
\text { network (ANN) }\end{array}$ \\
\hline 2 & $\begin{array}{l}\text { Manimala, } \\
\text { meghashree, } \\
\text { poornima gokhale } \\
\text { and sindhu } \\
\text { chandrashekhar }\end{array}$ & 2016 & $\begin{array}{l}\text { Image acquisition } \\
\text { and feature } \\
\text { extraction }\end{array}$ \\
\hline 3 & $\begin{array}{ll}\text { Prachi desai, } & \text { aayush } \\
\text { agarwal, } & \text { ajinkya } \\
\text { dhavale. } & \end{array}$ & 2015 & $\begin{array}{l}\text { Machine learning } \\
\text { approach (KNN } \\
\text { classifier). }\end{array}$ \\
\hline 4 & $\begin{array}{l}\text { Umang gupta, niladri } \\
\text { chatterjee }\end{array}$ & 2013 & $\begin{array}{l}\text { Rough set based } \\
\text { approach }\end{array}$ \\
\hline
\end{tabular}




\begin{tabular}{|c|c|c|c|}
\hline 5 & $\begin{array}{l}\text { Cleber Zanchettin, } \\
\text { Byron Leite Dantas } \\
\text { Bezerra et al }\end{array}$ & KNN-SVM \\
\hline 6 & $\begin{array}{l}\text { Champa HN and Dr } \\
\text { KR anandakumar }\end{array}$ & 2010 & ANN \\
\hline 7 & $\begin{array}{l}\text { Sargur N srihari, } \\
\text { sung-hyunk cha, hina } \\
\text { arora and sangjik lee }\end{array}$ & 2001 & ANN \\
\hline
\end{tabular}

Umang gupta, niladri chatterjee (2013) [5],explain the identification of personality trait by using rough set based machine learning method. As handwriting embedded with the human trait so in present work they use rough sets to extract the rules for prediction of human personality. They use ROSE2 tool for the comparison analysis in which firstly they extract the features of the written text, validate the data by using two methods basic minimal covering and satisfactory description and at last find the comparative values. This method include the improvement of neuroticism and extroversion whereas the SVM method had not observed sufficient improvements over what has been achieve using SVM based approach.

Cleber Zanchettin, Byron Leite Dantas Bezerra et al (2012) [6], presents the hybrid technique KNN-SVM for the recognition of cursive writing. Specialized support vector machines are introduced to increase the performance of KNN. The author used the different type of classifiers and database in experiment and proposed the hybrid technique. The recognition rate of handwritten text by using the hybrid technique of KNN-SVM has improved the performance in terms of recognition rate. So the experiment can improve he recognition rate from $1.00 \%$ to $3.61 \%$.in the end author can suggest the two future work proposals.

Sargur N srihari, sung-hyunk cha, hina arora and sangjik lee et al (2001) [7], has establish the individuality of the person by using machine learning method. The main goal of the author is to validate the individuality by comparing handwriting such as variability of handwriting of same person or the variability of handwriting from one person to another. The classification of Verification model can be done by using artificial neural network trained using backward error propagation with accuracy of $96 \%$ whereas identification model use nearest neighbor rule to classify the prototype document and using character level feature the performance measured was $98 \%$. The comparison of two methods can be done and they should make the conclusion with near $100 \%$ level of confidence.

Prof. Akshita Chanchlani, Pratima Kharade, Rutuja Kapase, Sonal Janvalka, Aakanksha Jaitly (2017) [8], proposed the method over graphologist in which they predict the behavior of human by taking parameters like slant, letter ' $\mathrm{i}$ ', ' $\mathrm{f}$ ', ' $\mathrm{a}$ ', ' $\mathrm{d}$ ', 'e', ' $g$ ' and so on. These parameters are input to the artificial neural network (ANN) which output the personality trait of human. The future work includes the recognition of cursive handwriting and hence proposed more robust system.

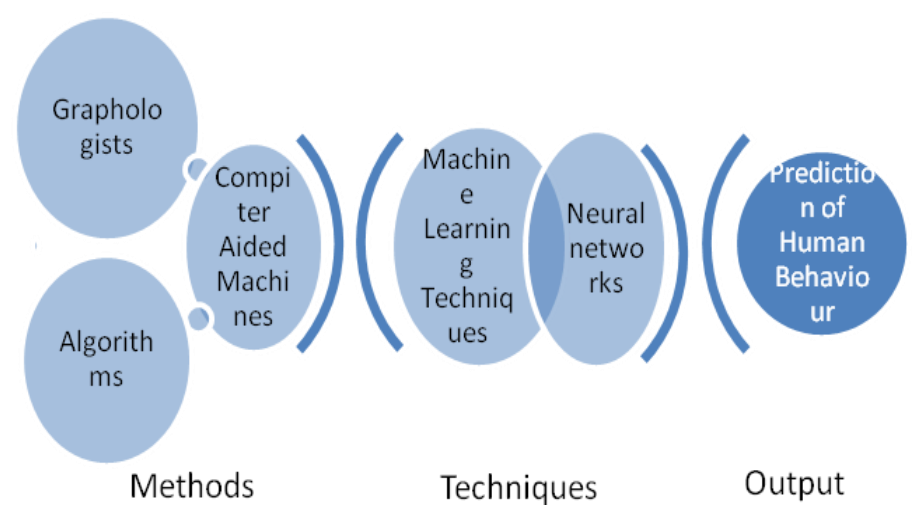

Figure 1: Methods and techniques used for predicting Human behavior.

\section{RESULT}

The result shows that handwriting can be recognized with new technology known as machine learning. The figures shows how machine learning work to recognize the alphabet.

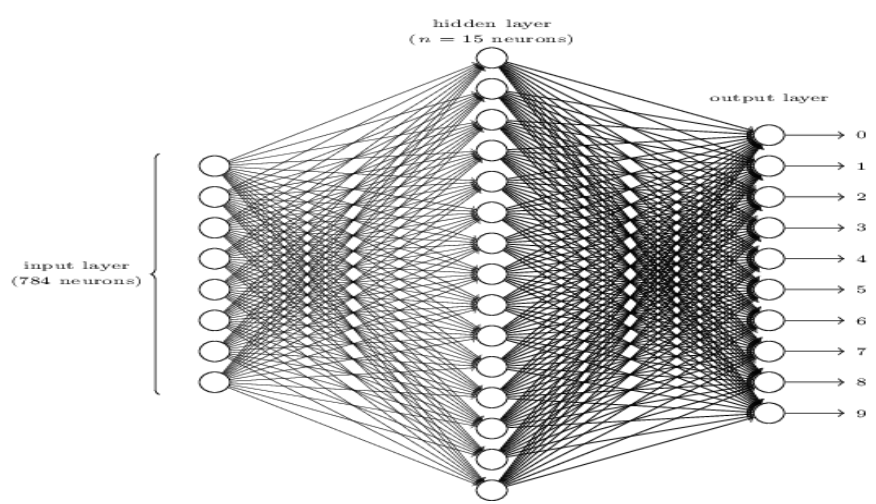

Figure 2: shows the working of Machine learning model 


\section{HANDWRITING RECOGNITION BY MACHINE LEARNING}

Figure 3: shows the simple model to recognize the alphabets.

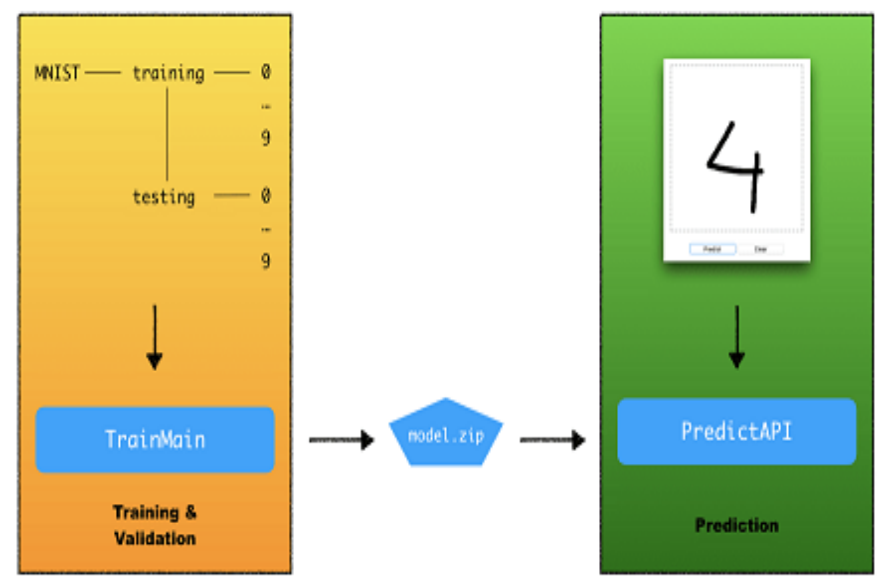

\section{CONCLUSION}

A detailed review of different authors has been described in this paper. Different methods and techniques used for the recognition of handwriting and hence predict the behavior of human has been explained here. The different parameters has taken under consideration are slant, margin, space between words, baseline, pen pressure and so on. These parameters are the input to the machine learning techniques such as artificial neural network, KNN-SVM, rough set based method and gives output in the form of human behavior. The future work can include one of the machine leaning technique used for analyze the behavior of human through handwriting with implementation and robust results.

\section{RESEARCH CONTRIBUTION}

Based on the literature reviewed, the paper focuses to build conceptual base and knowledge enhancement about the methods and techniques used in reviewing the human behavior. Major techniques used to recognize and predicting the human behavior are machine learning and neural networks. Further this research will help in determining human behavior patterns and personality traits of individual.

\section{REFERENCES}

1. Hemlata, Manoj Sachan, Shailendra Kumar Singh, "Personality Detection using Handwriting Analysis: Review ",The Seventh International Conference on Advances in Computing, Electronics and Communication - ACEC 2018.

2. Prachi desai, aayush agarwal, ajinkya dhavale, "Handwriting Analysis for Detection of Personality Traits using Machine Learning Approach", International Journal of Computer Applications (0975 - 8887), Volume 130 - No.15, November2015.

3. Champa HN and Dr KR anandakumar, "Artificial Neural Network for Human Behaviour Prediction through Handwriting Analysis, International Journal of Computer Applications (0975 - 8887)", Volume 2 - No.2, May 2010.

4. https://res.cloudinary.com/practicaldev/image/fetch/s --CXhG9fiS--

cc limit $\% 2 \mathrm{Cf}$ auto $\% 2 \mathrm{Cfl}$ progressive $\% 2 \mathrm{Cq} \_$auto $\% 2$ Cw 880/https://thepracticaldev.s3.amazonaws.com/i/ h368re5hbwf5sqnho0if.png

5. Manimala, meghashree, poornima gokhale and sindhu chandrashekhar, "AUTOMATED HANDWRITING ANALYSIS FOR HUMAN BEHAVIOR PREDICTION", International Journal of Computer Science and Engineering (IJCSE), Vol. 5, Issue 5, Aug - Sep 2016.

6. Umang gupta, niladri chatterjee, "Personality Traits Identification using Rough sets based Machine Learning”, International Symposium on Computational and Business Intelligence, 2013.

7. Cleber Zanchettin, Byron Leite Dantas Bezerra and Washington W. Azevedo, "A KNN-SVM Hybrid Model for Cursive Handwriting Recognition", WCCI 2012 IEEE World Congress on Computational Intelligence, June 10-15, 2012 - Brisbane, Australia.

8. Sargur N srihari, sung-hyunk cha, hina arora and sangjik lee, "Individuality of Handwriting: A Validation Study", 2001 IEEE.

9. Prof. Akshita Chanchlani1, Pratima Kharade, Rutuja Kapase, Sonal Janvalka, Aakanksha Jaitly, "International Journal for Research in Applied Science \& Engineering Technology (IJRASET)", Volume 5 Issue X, October 2017. 
HANDWRITING RECOGNITION BY MACHINE LEARNING

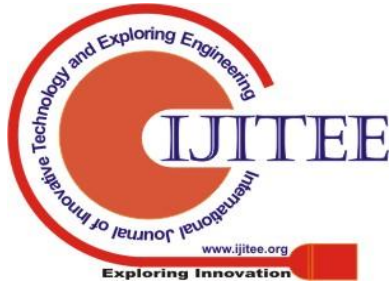

